\title{
Continuing the Mission of Pediatric Poison Prevention through Prescriber Education
}

\author{
Rebecca E. Bruccoleri ${ }^{1}$
}

Published online: 14 May 2015

(C) American College of Medical Toxicology 2015

Throughout fellowship, I have cared for countless young children who unintentionally ingested their parents', grandparents', or relatives' medications. While most patients do well, it is difficult to watch a child suffer the effects from the ingestion and witness the caregiver's guilt surrounding the event. For this reason, during every admission to our service for unintentional ingestions, we provide counseling to patients and families about medication safety during rounds and with our social workers. While prevention is a mainstay of the poison center mission for education and outreach, we continue to treat children with unintentional ingestions daily. An ideal solution to improve our medication safety education efforts should focus on adult providers and enlisting them to assist us in our poison prevention campaign. The first part of this educational mission is to make all prescribers (nurse practitioners, physician assistants, and physicians) aware of potentially dangerous medications to children. Teaching prescribers how to educate their patients on safe medication storage is the second part of this mission.

Many adult and specialty prescribers do not realize how dangerous one misplaced pill of a high-risk medication such as an opioid, sulfonylurea, tricyclic antidepressant, alpha-2 agonist, or calcium channel/beta-blocker can be for a child. A study by Burghardt et al. found significant associations with increased adult medication prescribing and increased pediatric exposures to opioids, oral hypoglycemics, anti-hyperlipidemics, and beta-blockers [1]. There was a stronger association with

Rebecca E. Bruccoleri

Rebecca.Bruccoleri@childrens.harvard.edu

1 Harvard Medical Toxicology Fellowship, Division of Emergency Medicine, Program in Medical Toxicology, Boston Children's Hospital, 300 Longwood Avenue, Boston, MA 02115, USA increases in opioid prescribing and exposures in the $0-5$-year age group. A $1 \%$ increase in adults receiving opioids was associated with 1.53 more exposures per 1,000,000 children between 0 and 5 years of age. In addition, the rate of hospitalizations was substantial for opioids (49.4\%) and oral hypoglycemics (35\%), some of the most commonly prescribed medications in the USA [1]. Tormoehlen et al. demonstrated increased adolescent opioid exposures reported to the Indiana Poison Center as the first or second exposure substance increased after the Joint Commission pain initiative took effect [2]. Spiller et al. showed an increase in pharmaceutical ingestions reported to five poison centers resulting in a serious medical outcome for patients less than 6 years of age between 2000 and 2010 [3]. Therefore, targeting and educating health-care providers on the importance of poison prevention efforts are a valuable strategy to help prevent unintentional pediatric ingestions.

As toxicologists, not only do we interact with many specialists such as hematologists, oncologists, obstetricians, gynecologists, and family practitioners, we also play a significant role in educating front-line prescribers about drug toxicity in high-risk patients. By taking advantage of these existing relationships, we can reiterate the importance of counseling patients on medication safety in person and during consultations. For example, when evaluating a parent who has children at home and then overdoses on a beta-blocker and recovers, part of the sign-off discussion with the team, patient, and family should include safe medication storage guidance or identifying safer antihypertensive medications that do not compromise patient care. Integrating discussions about medication safety into lectures for providers of all backgrounds (medical students, pharmacy students, medical and pharmacy residents, or medical and pharmacy fellows) is another means for improving provider knowledge and preparedness on the topic. 
Collaboration with pharmacists and pharmacy trainees is another helpful strategy on the wards and in retail where adult consumers obtain dangerous medications. Many hospitals have outpatient pharmacy services, and toxicologists could educate and encourage pharmacists to incorporate additional safe medication storage education into their counseling for high-risk medications. Another strategy is to encourage pharmacists to use flow-resistant bottles for liquid preparations. As many hospitals support pharmacy training programs and toxicologists are frequently involved in their education, there is a direct opportunity to educate pharmacy students and residents on the importance of counseling adults about pediatric poison prevention strategies.

There are already several existing efforts to diminish pediatric medication exposures and improve medication safety available to prescribers for additional guidance. The Up and Away campaign which is spearheaded by the PROTECT initiative through the $\mathrm{CDC}$ is another means to help prescribers educate their patients. When prescribing new medications, physicians can hand out pre-printed brochures available from Up and Away and counsel their patient on the importance on keeping prescription medications out of reach of their children. Alternatively, providers can refer patients to the AAPCC website to find more information about medication safety at www.aapcc.org. In the case of opioids prescribed for chronic pain or opiate use in the setting of addiction, naloxone is more frequently prescribed as a means to mitigate harm. There are multiple states involved in distribution of either free or thirdparty naloxone including North Carolina, Rhode Island, and Massachusetts [4-6]. When prescribed, education regarding naloxone use should include a discussion about use in all family members who are present in the home, not just the opioid user.

Patients do not take medications in a vacuum. By focusing education on avoiding inadvertent pediatric exposures, we can contribute to the safety of our patients' families and communities. As we are often called upon to share our expertise on adults with drug exposures or overdoses, we should seize this opportunity to discuss prescribed medications that present a significant risk to children in small quantities. We can be proactive with all prescribers and pharmacists to help educate patients receiving high-risk medications about unintentional ingestions. By taking advantage of existing relationships and daily interactions to educate prescribers, we can continue the mission of pediatric poisoning prevention and help protect our smallest patients.

Acknowledgments The author would like to thank Dr. Christine Murphy for all her assistance in preparing this manuscript.

\section{Sources of Funding None}

Conflict of Interest The author declares that she has no competing interests.

\section{References}

1. Burghardt LC, Ayers JW, Brownstein JS, Bronstein AC, Burns Ewald M, Bourgeois FT. Adult prescription drug use and pediatric medication exposures and poisonings. Pediatrics. 2013;132:18-26.

2. Spiller HA, Beuhler MC, Ryan ML, Borys DJ, Aleguas A, Bosse GM. Evaluation of changes in poisoning in young children: 2000 to 2010. Pediatr Emerg Care. 2013;29(5):635-40.

3. Tormoehlen LM, Mowry JB, Bodle JD, Rusyniak DE. Increased adolescent opioid use and complications reported to a poison control center following the 2000 JCAHO pain initiative. Clin Toxicol. 2011;49:492-8

4. Project Lazarus: Community-based overdose prevention and opioid safety with community care of North Carolina. http://projectlazarus.org/

5. Massachusetts Department of Public Health, Bureau of Substance Abuse Services. Opioid overdose response strategies in Massachusetts, April 2014. http://www.mass.gov/eohhs/docs/dph/substance-abuse/ opioid/overdoseresponsestrategies.pdf

6. Samuels E. Emergency department naloxone distribution: a Rhode Island Department of Health, recovery community, and emergency department partnership to reduce opioid overdose deaths. R I Med J. 2014;97:38-9. 